\title{
S-Adenosylmethionine in a Neuroblastoma
}

\author{
George M. Lyon, JR. [25] \\ Department of Pediatrics, Duke University Medical Center, Durham, North Carolina, USA
}

\begin{abstract}
Extract
A soluble extract of an abdominal neuroblastoma was assayed for catechol $O$-methyltransferase (COM) activity. No decrease in COM activity was found when $S$-adenosylmethionine was omitted from the reaction mixture. Extract of a metastatic, retro-orbital tumor from the same patient showed a strict dependence upon added $S$-adenosylmethionine for maximal stimulation. A deproteinized perchloric acid supernatant of the $15,000 \times g \times 30$-min tumor supernatant was placed on a Dowex-50-H+ column and eluted with sulfuric acid. A compound was found in the eluate which migrated as authentic $S$-adenosylmethionine in three different chromatographic systems. Visual comparison of the intensity of the unknown ninhydrin spot was made with known quantities of $S$-adenosylmethionine. The urine of the patient contained neither chromatographically detectable cystathionine nor elevated catecholamine catabolites.

The identity of $S$-adenosylmethionine as the unknown compound in the tumor was established by the following criteria: (1) ability to serve as a methyl donor in the COM reaction, (2) elution characteristics from a cation exchange resin, (3) migration in three different chromatographic solvent systems, (4) reactivity to ninhydrin, (5) reactivity to iodoplatinate, and (6) absorption of ultraviolet light.
\end{abstract}

\section{Speculation}

The metabolic basis for cystathioninuria in patients with neuroblastoma is not clear. The findings presented in this report may suggest that there is an association between catecholamine synthesis and degradation and the transsulfuration pathway. This could account for the observed intratumor accumulation of $S$-adenosylmethionine in the absence of catecholamine synthesis. Because of the diversity of biochemical processes utilizing $S$-adenosylmethionine many other explanations are equally tenable. The suggested model would predict, however, that there should be a correlation between urinary cystathionine excretion and the daily output of all catecholamine degradation products in patients with neuroblastoma.

\section{Introduction}

Urine from patients with neuroblastoma frequently contains abnormal or elevated amounts of metabolic substances presumably produced by the tumor. The most thoroughly described of these are the catecholamine degradation products [19]. Cystathionine may also be found in the urine $[6,7]$ as well as in the tumor tissue itself [8].
The data presented in this report describe chemical analyses upon neuroblastoma tumor tissue obtained from a patient who had neither detectable levels of cystathionine nor elevated catecholamine degradation products in his urine. The tumor, recovered surgically from his abdomen, contained high levels of a compound identified as $S$-adenosylmethionine. A metastatic, retro-orbital tumor, obtained later from the 
same patient, did not contain demonstrable amounts of the compound. The possible relation of these findings to the absence of cystathionine and catecholamine degradation products in the patient's urine is discussed.

\section{Materials and Methods}

Norepinephrine-7- ${ }^{3} \mathrm{H}, 8 \mathrm{Ci} / \mathrm{mmole}$ [20], and $S$-adenosylmethionine, $80 \%$ pure [21], were obtained commercially.

Abdominal and retro-orbital neuroblastoma tissue specimens were obtained at surgery, placed into an equal volume of $0.1 \mathrm{~m}$ Tris-HCl, $\mathrm{pH} 7.4$, minced into small fragments, and stored at $-10^{\circ}$ until used. Cell disruption was accomplished by sonication for $2 \mathrm{~min}$ at $4^{\circ}$. The sonicate was centrifuged at $15,000 \times g$ for $30 \mathrm{~min}$, and the supernatant was used as enzyme source.

Catechol $O$-methyltransferase ( $S$-adenosylmethionine: catechol O-methyltransferase) (EC. 2.1.1.a) activity was determined by the method of Axelrod [1]. The reaction mixture contained the $15,000 \times g$ supernatant extract of the tumor $(0.25 \mathrm{ml}, 100 \mathrm{mg}$ protein); norepinephrine-7-3 $\mathrm{H}, 25 \mu \mathrm{Ci}$ (5 m $\mu$ moles); magnesium chloride, $5 \mu$ moles; $S$-adenosylmethionine, $0.2 \mu$ mole;

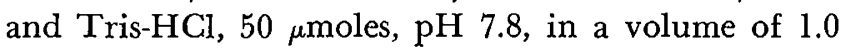
ml. Incubations were performed aerobically at $37^{\circ}$. In some experiments the total volume of the mixture was decreased, keeping the ratio of the reactants constant. Control tubes contained either boiled enzyme protein $\left(100^{\circ}, 5 \mathrm{~min}\right)$ or lacked added $S$-adenosylmethionine. Replicate, $0.2-\mathrm{ml}$ samples were withdrawn at the start of the incubation and at intervals thereafter, usually 30 and $60 \mathrm{~min}$. Each sample was immediately added to $1.0 \mathrm{ml}$ sodium borate, $0.1 \mathrm{M}, \mathrm{pH} 10.0$, and extracted with $5 \mathrm{ml}$ toluene-isoamyl alcohol $(3: 1, \mathrm{v}: \mathrm{v})$. Two milliliter samples of the organic phase of the extract were added to scintillation vials containing toluene, phosphor, and ethanol; the radioactivity was determined in a liquid scintillation counter.

In selected instances samples were obtained before the initiation of the incubation and spotted on Whatman no. 1 chromatography paper. Samples of the toluene-isoamyl alcohol extracts of the incubated reaction mixture were similarly spotted on chromatography paper and external and internal standards of nonradioisotopically labeled norepinephrine and normetanephrine were included in the chromatographic runs. The chromatograms were developed, ascending, in bu- tanol-ethanol-water $(4: 1: 1, \mathrm{v}: \mathrm{v}: \mathrm{v})$ (solvent I), butanol-acetic acid-water $(4: 1: 1, \mathrm{v}: \mathrm{v}: \mathrm{v})$ (solvent II), or $n$ propanol-ammonia $(1.4 \mathrm{~N})(4: 1, \mathrm{v}: \mathrm{v})$ (solvent III). The standards were localized by spraying the dried chromatograms with freshly diazotized $p$-nitroaniline followed by $1 \mathrm{~m}$ potassium carbonate. After drying, each chromatographic lane was cut into equal sequential sections and placed into individual scintillation vials containing toluene and phosphor; the radioactivity of each section was determined.

The $S$-adenosylmethionine content of the $15,000 \times g$ tumor supernatants was determined by first making the sonicated tumor supernatants $1 \mathrm{~N}$ with respect to perchloric acid at $4^{\circ}$. The precipitate was sedimented by centrifugation, and the deproteinized supernatant was passed through a Dowex- $50-\mathrm{H}+$ column $(1.0 \mathrm{~cm} \times$ $10.0 \mathrm{~cm})$. The column was sequentially eluted with $1 \mathrm{~N}$ $(100 \mathrm{ml}), 2 \mathrm{~N}(100 \mathrm{ml})$, and finally $6 \mathrm{~N}$ sulfuric acid $(50$ ml) [12]. The material eluting with $6 \mathrm{~N}$ sulfuric acid was collected, pooled, and neutralized by the addition of barium carbonate. The precipitated barium sulfate was removed by centrifugation, and the supernatant was reduced in volume, in vacuo.

Samples of the neutralized column eluate were placed on Whatman no. I paper and chromatographed, ascending, in a variety of solvents. These included $t$-butanol-methyl ethyl ketone-water-ammonium hydroxide $(4: 3: 2: 1)$ (solvent IV), and $n$-butanol-glacial acetic acid-water $(2: 1: 1, \mathrm{v}: \mathrm{v}: \mathrm{v})$ (solvent V). Authentic, $80 \%$ pure $S$-adenosylmethionine was used as an external standard. Cellulose thin layer chromatography of the eluate was carried out in solvent I. The plates were scanned with ultraviolet light [22] (principal emission, $254 \mathrm{~m}_{\mu}$ ), and stained with either iodoplatinic acid reagent [18], cyanide-nitroprusside reagent [14], or ninhydrin.

Concentration of 3-methoxy-4-hydroxymandelic acid (VMA) in urine was determined using the method of Pisano et al. [11]. Chromatographic analysis of the urine failed to detect homovanillic acid, a compound known to decrease the sensitivity of the Pisano test [2]. All protein determinations were by the method of Lowry et al. [10].

Ninhydrin-positive material in the urine of the patient was analyzed by two-dimensional chromatography in pyridine-acetone-ammonia-water $(4: 1: 1: 1$, v:v:v:v) (solvent VI), followed by isopropanol-formic acid-water $(2: 1: 1, \mathrm{v}: \mathrm{v}: \mathrm{v})$ (solvent VII), or by electrophoresis followed by chromatography after the method of Stevens [16]. 


\section{Results}

\section{Catechol O-Methyltransferase (COM) Activity}

The soluble extract of the abdominal tumor was assayed for COM activity. Figure 1 shows the time course for the conversion of radioactively labeled norepinephrine to organically extractable material. Alterations in either substrate concentrations or amount of added enzyme extract did not improve the linearity of the reaction with respect to time. Figure 2 shows the distribution of radioactivity in the reaction mixture at the start of the incubation $(A)$ and in the organic phase of the extract following a 60 -min incubation $(B)$. The chromatograms were developed, ascending, in solvent $\mathrm{I}$. The major peaks of radioactivity corresponded, respectively, to authentic norepinephrine $(A)$ and normetanephrine $(B)$.

An incubation time of $30 \mathrm{~min}$ was chosen as an optimum for determination of enzymatic activity. Table I shows the amount of organically extractible radioactivity present after a 30 -min incubation of extracts with norepinephrine-7-3 $\mathrm{H}$ under varying conditions. When the abdominal tumor extract was used, there was no difference between the complete reaction mixture and the control sample which lacked added $S$-adenosylmethionine. Extract of the metastatic, retroorbital tumor showed a strict dependence upon added

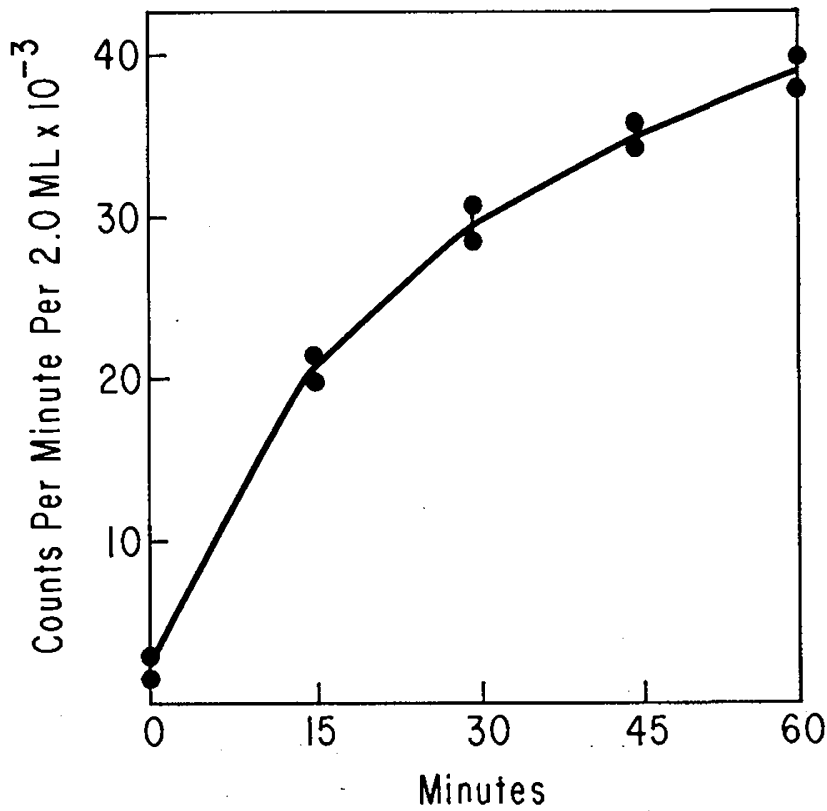

Fig. 1. Appearance of organically extractible radioactivity as a function of time. The incubation mixture contained abdominal tumor extract as catechol $O$-methyltransferase source and the incubation was carried out as described in Methods.

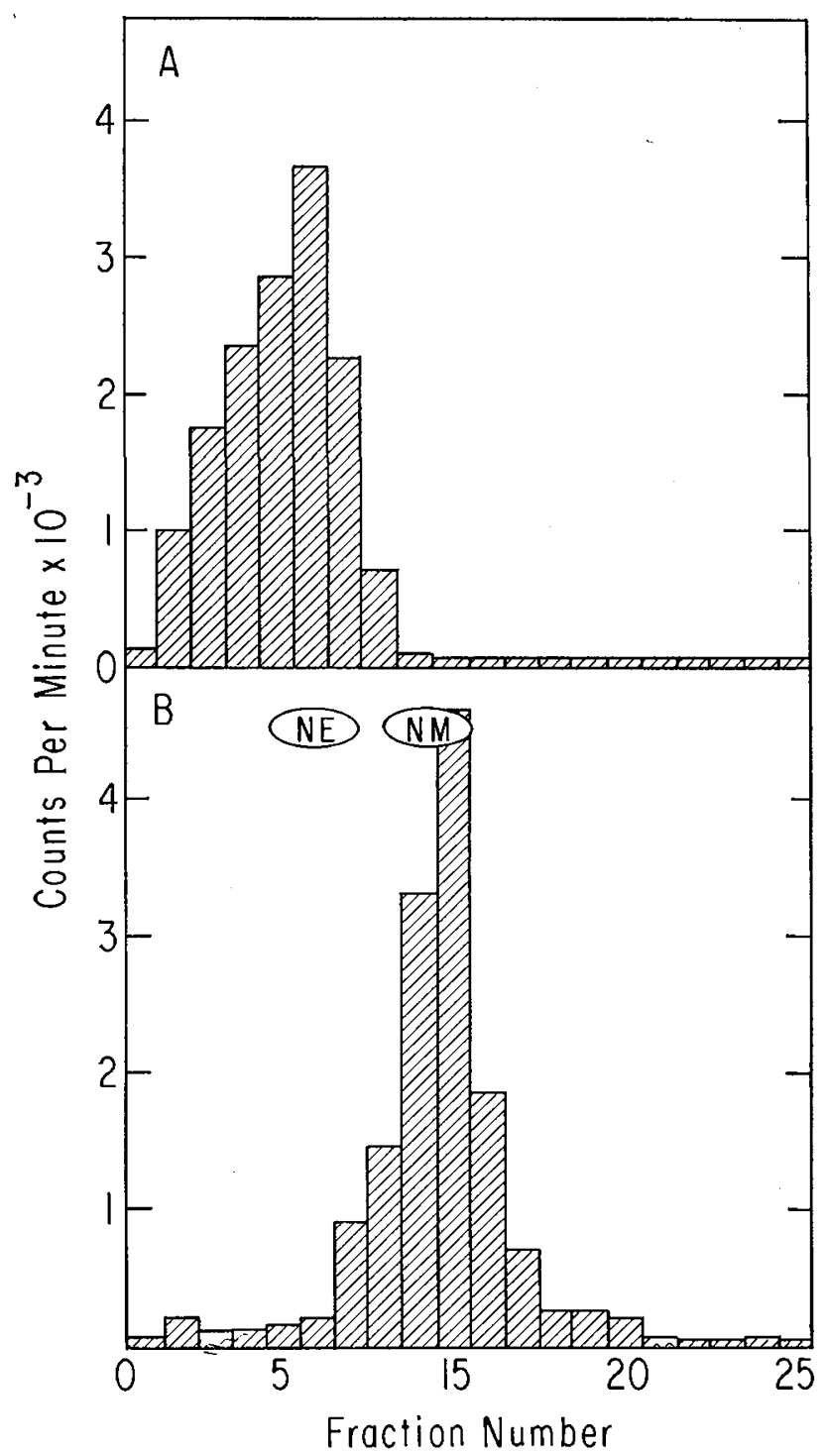

Fig. $2 A \& B$. Chromatographic distribution of radioactivity at the start of the incubation $(A)$ and as found in the toluene-isoamyl alcohol extract after $60 \mathrm{~min}(B)$. Chromatography was carried out in solvent $I$ and the distribution of the radioactivity was determined as described in Methods. The origin was at fraction 1 and the solvent front was at fraction 25. The closed circles represent the migration of authentic norepinephrine (NE) and normetanephrine (NM) in this system. The substrate was DLnorepinephrine-7-3 $\mathrm{H}$ and abdominal tumor extract was used as catechol $O$-methyltransferase source.

$S$-adenosylmethionine for enzymatic activity. Overnight dialysis of the abdominal tumor extract, at $4^{\circ}$, resulted in a dependence of activity upon added $S$-adenosylmethionine. Addition of $S$-adenosylmethionine to the dialyzed sample resulted in recovery of approximately one-half of the activity originally present. This may result from inactivation of enzyme by the dialysis 
Table I. Catechol $O$-methyltransferase activity of abdominal and retro-orbital neuroblastoma tissue

\begin{tabular}{llc}
\hline \multicolumn{1}{c}{ Enzyme source } & \multicolumn{1}{c}{ Conditions } & $\begin{array}{c}\mathrm{cpm} / 2.0 \mathrm{ml} \\
\text { extract }\end{array}$ \\
\hline TD-1 (abdominal & Boiled & 10,980 \\
tumor) & & 10,040 \\
& Less $S$-adenosylmethio- & 42,500 \\
& nine & 41,980 \\
& Complete & 42,500 \\
& & 43,400 \\
TD-1 (dialyzed) & Boiled & 11,390 \\
& & 11,070 \\
& Less $S$-adenosylmethio- & 13,060 \\
& nine & 13,200 \\
& Complete & 28,620 \\
& & 28,570 \\
TD-2 (retro-orbital & Boiled & 11,200 \\
tumor) & & 10,710 \\
& Less $S$-adenosylmethio- & 13,960 \\
& nine & 12,390 \\
& Complete & 44,150 \\
& & 41,690
\end{tabular}

procedure. Rapid passage of the enzyme extract through a Sephadex G-25 coarse column resulted in a recovery of $90 \%$ of activity which was dependent upon added $S$-adenosylmethionine.

Figure 3 shows chromatographic analyses of the organically extractible radioactivity found with and without addition of $S$-adenosylmethionine to the reaction mixture containing nondialyzed extracts of the abdominal tumor protein. The peaks of radioactivity migrated identically to authentic normetanephrine in three different solvent systems (I, II, and III), demonstrating that in each reaction normetanephrine was formed.

\section{S-Adenosylmethionine in the Tumor Extract}

A deproteinized perchloric acid supernatant of the $15,000 \times g$ tumor supernatant was placed on a Dowex-50-H+ column and eluted with sulfuric acid. The material eluting with $6 \mathrm{~N}$ sulfuric acid from the column was neutralized with solid barium carbonate and reduced in volume, in vacuo. Unfortunately, this procedure causes degradation of $20-30 \%$ of the $S$-adenosylmethionine [12]; consequently, the estimation of the initial concentration of the compound in the tumor is a minimal value. Attempts to recover $S$-adenosylmethionine as either the Reinecke salt [3] or phosphotungstic acid precipitate [15] were not successful, possibly because of the large eluate volume containing a small amount of the $S$-adenosylmethionine (Fig. 4).

Ninhydrin-positive material present in the neutral- ized column eluates migrated identically to the major spot found when $80 \%$ pure $S$-adenosylmethionine was chromatographed in solvents IV and V. The $R_{F}$ values for the unknown spot were 0.33 in solvent IV and 0.16 in solvent $\mathrm{V}$, which corresponded to established values of 0.35 and 0.17 , respectively [4]. This spot absorbed ultraviolet light, was ninhydrin-positive, gave a clear spot with the iodoplatinic acid reaction, and was negative to cyanide-nitroprusside.

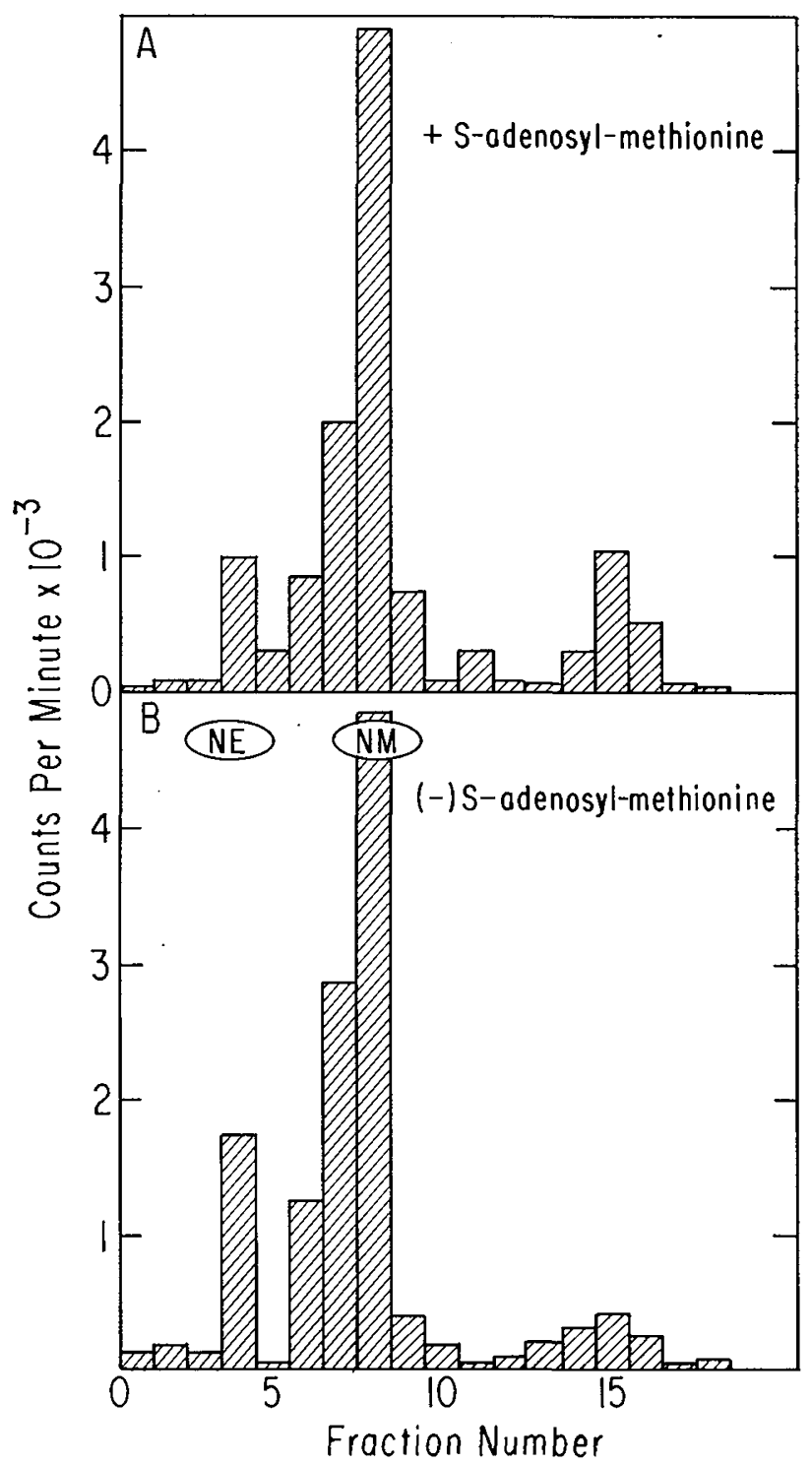

Fig. $3 A \& B$. Chromatographic distribution of organically extractible radioactivity following a $30-\mathrm{min}$ incubation in the presence $(A)$ and absence $(B)$ of added $S$-adenosylmethionine. Enzyme source was abdominal tumor extract and the incubations were carried out as described in Methods. The solvent front was fraction 18 and the migration of norepinephrine (NE) and normetanephrine (NM) in this system (solvent $\mathrm{I}$ ) are shown. 


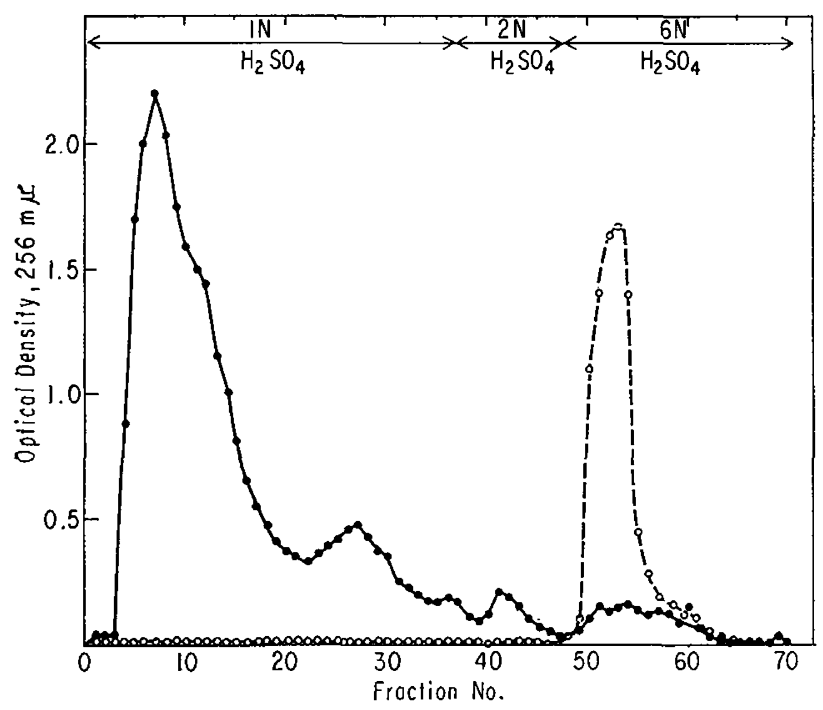

Fig. 4. Elution of deproteinized abdominal tumor supernatant from Dowex-50- $\mathrm{H}^{+}(-\bullet)$ as described in Methods. (O-O): elution pattern of authentic, $80 \%$ pure $S$-adenosylmethionine from an identical column. The material eluting in the first 50 $\mathrm{ml} 6 \mathrm{~N}$ sulfuric acid was pooled and treated as described in Methods.

Judged by the intensity of the ninhydrin spot of the unknown, a rough quantitation of the amount of $S$-adenosylmethionine can be made, but, because of breakdown during isolation, quantitation of this sample can only provide a number representative of a minimal concentration initially present in the tumor. Visible comparison of the intensity of the unknown ninhydrin spot with known quantities of $S$-adenosylmethionine suggests that the original tumor contained approximately $0.4 \mu$ mole $S$-adenosylmethionine/g wet weight tissue. Elution of the main ultraviolet-absorbing spot on the chromatogram with water and subsequent determination of the optical density of the solution at $256 \mathrm{~m} \mu$ provide a minimal value of $0.2 \mu$ mole $S$-adenosylmethionine/g wet weight of tissue. This is based upon a millimolar extinction for $S$-adenosylmethionine of $15,000\left(E_{256 \mathrm{~m} \mu}^{10^{-3} \mathrm{M}}=15,000\right)$ [3].

Neither cystathionine nor homocysteine was detectable by ninhydrin following two-dimensional thin layer chromatography of $0.05-\mathrm{ml}$ samples of the patient's urine. The most prominent ninhydrin-positive spot coincided with the migration of $\beta$-aminoisobutyric acid.

\section{Discussion}

Cystathioninuria in patients with neuroblastoma [7] and hepatoblastoma [5, 9] is well documented. The patient described in this report, although having a neuroblastoma, did not elaborate detectable quantities of cystathionine or have elevated catecholamine degradation products in his urine. Furthermore, the abdominal tumor did not contain detectable amounts of cystathionine when a protein-free supernatant of the tumor was examined by two-dimensional thin layer chromatography and ninhydrin spray. Extracts of the tumor contained $S$-adenosylmethionine at a concentration of $0.2-0.4 \mu \mathrm{mole} / \mathrm{g}$ wet weight tumor. A metastatic tumor from the same patient failed to demonstrate similar levels of $S$-adenosylmethionine.

The identity of $S$-adenosylmethionine in the tumor is established by its ( $I$ ) function as a methyl donor in the COM reaction, (2) elution characteristics from a cation exchange resin, (3) migration in three different chromatographic solvent systems (solvents I, IV, V), (4) reactivity to ninhydrin, (5) reactivity to iodoplatinate, and (6) absorption of ultraviolet light. Because of the small quantity of material available elemental analysis could not be carried out.

To our knowledge, $S$-adenosylmethionine has not been reported in tumor tissue at the concentrations found in the tumor of this patient. Since in the urine of this patient the usual findings of neuroblastoma, i.e., increased methylated catecholamine derivatives and cystathioninuria, were not apparent, this may represent an unusual tumor variation.

Clearly, since $S$-adenosylmethionine is involved in a vast number of reactions, many explanations for the accumulation of the compound in this tumor are possible. A simple, though speculative, explanation is as follows. Many neuroblastomas are capable of elaborating large amounts of catecholamines that require equivalent amounts of methyl donor, $S$-adenosylmethionine, for pharmacological inactivation. A priori, in addition to catecholamine synthesis the transsulfuration pathway must be present and equally active and cystathionine may represent the urinary end product of this pathway. Those few cases of neuroblastoma which present with diarrhea, labile hypertension, and elevated, circulating pressor amines [17] could be special cases where catecholamine synthesis continues but there is decreased availability of methyl donor leading to secretion of pressor amines. The converse situation, inability to synthesize catecholamines, might present as the tumor described in this report. The tumor retains the ability to synthesize large quantities of methyl donor, a condition which may be analogous to derepression of the pathway, but, in the absence of large amounts of methyl acceptor (catecholamines), there is an intracellular accumulation of $S$-adenosylmethionine.

We are currently attempting to determine whether there is a correlation between daily cystathionine ex- 
cretion and the amount of catecholamine degradation products in the urines of our patients with neuroblastoma. To date, insufficient data have been accumulated to test the validity of the hypothesis. In the study of Geiser and Efron [6] no correlation was evident. These authors quantitated cystathionine and VMA in urine. In Gjessing's series [7] a better correlation was found, perhaps because he quantitated homovanillic acid in addition to VMA and cystathionine. The presence of cystathioninuria in patients with hepatoblastomas may weaken this argument although a similar mechanism, with increased $S$-adenosylmethionine utilization, may be evoked, assuming there is need for large amounts of methylation of other compounds, not necessarily catecholamines.

The lack of $S$-adenosylmethionine in the metastatic tumor is difficult to explain. In the period of time between recovery of the tissues the retro-orbital lesion had received extensive radiation therapy and the patient had been on systemic chemotherapy with cyclophosphamide. Thus, whether this difference is secondary to treatment or due to a molecular alteration in the metastatic tumor is not clear.

Further investigations are being carried out in our laboratory to determine whether there is a correlation between total catecholamine degradation and cystathionine excretion in these patients, and all tumor material is being analyzed for $S$-adenosylmethionine, cystathionine, and COM activity.

\section{Summary}

Tissue derived from an abdominal neuroblastoma contained a high level of catechol $O$-methyltransferase activity which was not dependent upon addition of the methyl donor, $S$-adenosylmethionine, for maximal activity. Analysis of the tumor extract detected a compound which chromatographically and chemically was identical to authentic $S$-adenosylmethionine. Using chromatographic techniques, neither cystathionine nor elevated catecholamine catabolites were found in the urine of the patient.

\section{References and Notes}

1. Axelrod, J.: Catechol $O$-methyltransferase from rat liver. In: S. Colowick and N. Kaplan: Methods in Enzymology, Vol. V, p. 748 (Academic Press, New York, 1962).

2. Boнoun, C.: Catecholamine metabolism in neuroblastoma. J. Pediat. Surg., 3: 114 (1968).

3. Cantoni, G. L.: Preparation of $S$-adenosylmethionine. In: S. Colowick and N. Kaplan: Methods in Enzymology, Vol. III, p. 600 (Academic Press, New York, 1957).

4. Fink, K., AND Adams, W. S.: Paper chromatographic data for purines, pyrimidines, and derivatives in a variety of solvents. J. Chromatogr., 22: 118 (1966).

5. Geiser, C. F., Baez, A., Schindler, A. M., and Shih, V. E.: Epithelial hepatoblastoma associated with congenital hemihypertrophy and cystathioninuria. Presentation of a case. Pediatrics, 46: 66 (1970).

6. GeIser, C. F., ANd EFron, M. L.: Cystathioninuria in patients with neuroblastoma or ganglioneuroblastoma. Cancer, 22: 856 (1968).

7. GJEssing, L. R.: Studies of functional neural tumors. II. Cystathioninuria. Scand. J. Clin. Lab. Invest., 15: 474 (1963).

8. GJEssing, L. R.: Studies on functional neural tumors. III. Cystathionine in the tumor tissue. Scand. J. Clin. Lab. Invest., 15: 479 (1963).

9. Gjessing, L. R., AND Mauritzen, K.: Cystathioninuria in hepatoblastoma. Scand. J. Clin. Lab. Invest., 17: 513 (1965).

10. Lowry, O., Rosebrough, N., Farr, A., and Randall, R.: Protein measurement with the Folin phenol reagent. J. Biol. Chem. 193: 265 (1951).

11. Pisano, J. J., Croux, J. R., and Abraham, D.: Determination of 3-methoxy-4-hydroxymandelic acid in urine. Clin. Chim. Acta, 7: 285 (1962).

12. Schlenk, F., Danko, J. L., and Stanford, S. M.: Improved procedure for the isolation of $S$-adenosylmethionine and $S$ adenosylhomocysteine. Arch. Biochem. Biophys., 83: 28 (1959).

13. SCHWEISGUTH, O.: Excretion of catecholamine metabolites in urine of neuroblastoma patients. J. Pediat. Surg., 3: 118 (1968).

14. Smirh, I.: Aminoacids, Amines, and Related Compounds; Chromatographic and Electrophoretic Techniques, Vol. I, p. 82 (Interscience Publishers, New York, 1960).

15. Stekol, J. A., Anderson, E. I., and Weiss, S.: $S$-Adenosyl-tmethionine in the synthesis of choline, creatine, and cysteine in vivo and in vitro. J. Biol. Chem., 233: 425 (1958).

16. Sxevens, K. M.: Amino acid electrophoresis. In: S. T. Nerenberg: Electrophoresis, p. 147 (F. A. Davis, Philadelphia, 1966).

17. Stickler, G. B., AND Flock, E. V.: Neuroblastoma and ganglioneuroblastoma: Associated increased urinary excretion of catecholamines. Cancer Chemother. Rep., 16: 439 (1962).

18. Toennies, G., and Kalb, J. J.: Techniques and reagents for paper chromatography. Anal. Chem., 23: 823 (1951).

19. Von Studnitz, W., Kaser, H., and SJoerdsma, A.: Spectrum of catecholamine biochemistry in patients with neuroblastoma. New Engl. J. Med., 269: 232 (1963).

20. New England Nuclear Corporation, Boston, Mass.

21. Sigma Chemical Company, St. Louis, Mo.

22. Mineralight, Scientific Products, Evanston, Ill.

23. The author wishes to thank Mr. Mason Brooks for his expert technical assistance and Dr. F. S. Porter and Dr. J. B. Sidbury for their interests and criticism.

24. Supported by the United Medical Research Fund of North Carolina, the Research Fund of the Duke Endowment, and National Cancer Institute Research Career Development Award no. 1-K04-CA-20, 487-01. Portions of this study presented to the Society for Pediatric Research, Atlantic City, N. J., May 2, 1970.

25. Requests for reprints should be addressed to: George $M$. Lyon, Jr., M.D., Department of Pediatrics, Duke University Medical Center, Durham, N. C. 27706 (USA).

26. Accepted for publication October 12, 1970. 